\title{
Analysis of Serum Biochemical Indexes for the Diagnosis of Coronary Heart Disease in Suspected Patients
}

\author{
Hongcheng Mai ${ }^{*}$, Zhifeng Huang2*, Tao Zhang3\# \\ ${ }^{1}$ Department of Internal Neurology, Guangzhou Overseas Chinese Hospital, The First Affiliated Hospital of \\ Jinan University, Guangzhou, China \\ ${ }^{2}$ Medical College of Jinan University, Guangzhou, China \\ ${ }^{3}$ Department of Cardiology, Guangzhou Overseas Chinese Hospital, The First Affiliated Hospital of Jinan \\ University, Guangzhou, China \\ Email: "heartdisease@126.com
}

Received 12 February 2016; accepted 27 February 2016; published 2 March 2016

Copyright (C) 2016 by authors and OALib.

This work is licensed under the Creative Commons Attribution International License (CC BY).

http://creativecommons.org/licenses/by/4.0/

(c) (i) Open Access

\begin{abstract}
Background: The serum biochemical indexes are classic detection in clinical practice. Methods: In this study, for avoiding the risks of serious complications, high-cost in diagnosis of suspected coronary heart disease (CHD), the common biochemical indexes were detected from 68 eligible patients with suspected CHD. Treadmill exercises test (TET)-electrocardiogram (ECG) was measured during TET, and invasive examination of coronary angiography (CAG, golden standard for CHD diagnosis) was also performed. Results: For CAG, 48 patients were positive and 20 were negative; while for TET 38 were positive, 14 were inconclusive, and 16 were negative, respectively. Among these biochemical indexes, the HbA1c (\%) level in CAG positive patients was much higher than that in CAG negative patients $(P=0.019)$. Furthermore, according to receiver operating characteristic (ROC) curve, HbA1c showed effective diagnosis for CHD and its best cutoff value was $5.85 \%$ and 29 of the patients were HbA1c positive and 30 were negative. Conclusions: It was found that HbA1c combined with TET obviously enhanced the sensitivity of examinations. All the patients who were negative in both HbA1c and TET tests turned out to be $90 \%$ CAG negative, which meant that the combination might stand invasive examination of CAG for CHD diagnosis. Further studies in multi-center investigation will be expected to validate the findings.
\end{abstract}

\section{Keywords}

Treadmill Exercise Test, Coronary Angiography, Receiver Operating Characteristic, Glycated Hemoglobin, Coronary Heart Disease, Low-Density Protein

\footnotetext{
*These authors contributed equally to this work.

${ }^{\#}$ Corresponding author.
} 


\section{Introduction}

CHD has a close relationship with myocardial ischemic disease that threatens patients' life. Various risk factors are associated with CHD, such as diabetes, severe hypertension and hypercholesterolemia. Diabetes is one of the major causes of CHD and nearly 70\% type 2 diabetes die of cardiovascular causes, especially CHD. Moreover HbA1c was associated with a more obvious increase in diabetes [1].

Coronary angiography (CAG) is the "gold standard" for myocardial ischemia diagnosis. However, as CAG is only cost-effectively conducted in highly suspected patients, it has the risks of serious complications and might be impossible in some common hospitals and affect the diagnosis of CHD in some patients [2].

As the non-invasive detective way, ECG measured during TET (TET-ECG) is a more sensitive way of identifying CHD. TET can give extra load to heart so that oxygen consumption of myocardium increases, which can arouse ischemia in potential cases before inhibition of glyceryl trinitrate [3], meanwhile ECG abnormalities can be recorded in terms of "ST-T changes" by synchronous 12-lead ECG. Another non-invasive method is blood test. The classic biochemical indexes of common detection have been used as diagnostic tools of acute myocardial infarction and other potential causes in clinical practice. But the efficient serum biochemical index combined TET to detect coronary heart disease is not found in suspected patients. So it is necessary to find effective diagnostic biochemical indexes alone or combined with TET to confirm the suspected CHD patients to promote the effective diagnosis rate.

In this study, TET results and biochemical indexes of all the 68 patients were recruited for the inconclusive TET diagnosis. For all these examinations, CAG is the "gold standard" to make a definite CHD diagnosis, and TET combined with other indexes can improve the diagnostic effect of CHD.

\section{Materials and Methods}

\subsection{Patients and General Procedures}

68 patients with complaints of "chest pressure, chest pain, or chest discomfort" [4] were enrolled in the First Affiliated Hospital of Jinan University (Guangzhou, China) from January 2012 to August 2014 for suspected CHD. All the patients of peripheral artery disease, renal function insufficiency, myocardial infarction and nonclinical indications for testing like post-heart transplant evaluation or participation in a research protocol were excluded before TET [5]. All the patients were confirmed diagnosis by carrying out of CAG after TET within one month. The First Affiliated Hospital of Jinan University approved this study.

\subsection{Materials}

Biomarker were analysed by HITACHI-7600 automatic biochemistry analyzer (HITACHI, Japan); TET was analysed by GE Cardiosoft V6.51 system (Mortara Instrument, USA); CAG was conducted by GE innova 3100 X-ray machine (General Electric Company, USA).

\subsection{TET}

TET adopted Bruce scheme, which the patients should reach 100\% (maximal exercises) or 85\% (submaximal exercise) of their maximal heart rate (220 age). After the Bruce protocol, the Duke Treadmill Score was calculated as following: exercise time (ET, min) - (5×maximal ST-segment depression [mm] $)-(4 \times$ angina index [no Angina, 0; Angina, 1; Angina as reason for stopping test 2]) [6].

The "positive" criteria were: ECG showed upsloping ST-segment depressions $\geq 1.5 \mathrm{~mm}$ or downsloping or horizontal depressions $\geq 1.0 \mathrm{~mm}$ in at least 2 leads and last for more than $2 \mathrm{~min}$, with or without concomitant typical angina symptoms; while the "negative" criteria were: objective load achieved without ST-T changes [7].

\subsection{Coronary Angiography}

All 68 patients were tested CAG that Seldinger technique was adopted to puncture and a cather was carefully 
moved to heart before dye injecting. Stenosis or any blokages in blood flow can be highlighted by dye moving through the artery. At least a stenosis $\geq 50 \%$ in the coronary artery was defined as CHD [8].

\subsection{Data Analysis}

Data were presented as mean \pm SEM. For baseline characteristics of between CAG positive and negative were tested by Chi-square test. For biochemical indexes mean comparisons between TET positive and negative were tested by independent samples $t$-test. Receiver operating characteristic (ROC) curve was used for general evaluation of biochemical indexes. Calculation and illustration were made by software SPSS 13.0. Statistical significance was established at $P<0.05$.

\section{Results}

\subsection{Baseline Characteristics and Exercise Parameters Results}

Table 1 depicted baseline characteristics and exercise parameters of the 68 patients (Age: $59 \pm 1.1$ years, 66\% male) who participated in CAG and TET. In comparison to the baseline characteristics and exercise parameters of the negative $(-)$ and positive (+) CAG patients, they were considered positive as at least $50 \%$ stenosis. An analysis showed that there were higher evaluations in Duke Treadmill Score (Chi-square test, $P<0.001$ ), Hypertenstion (Chi-square test, $P=0.034$ ), and Diabetes (Chi-square test, $P=0.024$ ) among them.

The analysis also showed that positive CAG patients compared to negative CAG patients had higher evaluations in peak heart rate (Peak HR, bmp) (Independent $t$-test, $P<0.05$ ), maximum peak heart rate achieved (MPHR achieved \%) (Independent $t$-test, $P<0.05$ ) and maximum RPP (Independent $t$-test, $P<0.05$ ). Other baseline characteristics and exercise parameters had no significantly different between positive and negative CAG patients $(P>0.05)$.

\subsection{Determinations and Tests of Biochemical Indexes}

As shown in Table 2, among detected serum biochemical indexes, HbA1c (glycosylated hemoglobin, \%) values in CAG positive group were much higher than those in negative group, and the difference of HbA1c between positive and negative CAG had statistic significance $(P=0.041)$. In other words, HbA1c values were statistically different between CHD patients and non-CHD persons in TET positive group.

\subsection{ROC Curve of HbA1c for CHD Diagnosis}

ROC curve was often used to determine the best cutoff value for a diagnostic test. The Test Variable was defined as new variable, and the State Variable was defined as the results of "golden diagnosis", a series of sensitivity and specificity values can be calculated. The ROC curve was made with sensitivity (the true positive rate) as $y$-axis and 1-specificity (the false positive rate) as $\mathrm{x}$-axis, to show the relationship between sensitivity and specificity. ROC curve which is shaped with convexity towards the upper-left quadrant, where the point most close to was chosen as the best cutoff value, of the reference frame was desirable [7]. On the other hand, the area under the curve (AUC) can be done to identify the ability of the test as a diagnostic tool. Then with the results of CAG as "gold standard" for CHD, there was statistic significance of HbA1c as a diagnostic biomarker for CHD [AUC $=0.718(0.500,0.867), P<0.05]$, and we got the best cutoff value of $5.85 \%$ according to the curve (Figure 1).

\subsection{Examinations of CAG and TET}

All patients were executed TET and CAG. As for CAG, "positive" was defined as existing at least a stenosis $\geq$ $50 \%$ in the coronary artery. In Table 3, 48 (70.6\%) were positive and 20 (29.4\%) were negative. Among the 68 studied TET patients, 38 (55.9\%) were positive, 14 (20.6\%) were inconclusive, and 16 (23.5\%) were negative. Among the 68 studied patients, 59 were detected HbA1c value, 29 (49\%) were positive, 30 (51\%) were negative.

\subsection{Different Detections for CHD Diagnosis}

Taking CAG results as gold standard for CHD diagnosis, we compared four different detections for CHD diag- 
Table 1. Baseline characteristics stratified by CAD patients.

\begin{tabular}{|c|c|c|c|c|}
\hline & All $(\mathrm{n}=68)$ & Negative CAG $(n=20)$ & Positive CAG $(n=48)$ & $p$ \\
\hline Male gender, $\mathrm{n}(\%)^{\#}$ & $45(66)$ & $11(55)$ & $34(71)$ & 0.214 \\
\hline Age (y) & $59 \pm 1.1$ & $59 \pm 1.9$ & $59 \pm 1.3$ & 0.979 \\
\hline Hypertension, $\mathrm{n}(\%)^{\#}$ & $34(50)$ & $6(30)$ & $28(58)$ & 0.034 \\
\hline Diabetes, n (\%) & $24(35)$ & $3(15)$ & $21(44)$ & 0.024 \\
\hline Hyperlipoidemia, n (\%) & $19(28)$ & $6(30)$ & $13(27)$ & 0.811 \\
\hline Smoking, $\mathrm{n}(\%)^{\#}$ & $23(34)$ & $8(40)$ & $15(31)$ & 0.494 \\
\hline $\begin{array}{c}\text { Family History of CAD, n }(\%)^{\#} \\
\text { pre-TET diagnosis }\end{array}$ & $8(12)$ & $3(15)$ & $5(10)$ & 0.599 \\
\hline Asymptomatic (\%) ${ }^{\#}$ & $28(41)$ & $8(40)$ & $20(42)$ & 0.901 \\
\hline After PCI (\%) $)^{@, \#}$ & $12(18)$ & $1(5)$ & $11(23)$ & 0.08 \\
\hline Symptomatic (\%) ${ }^{\#}$ & $29(43)$ & $11(55)$ & $18(38)$ & 0.189 \\
\hline MET & $8.385 \pm 0.191$ & $8.650 \pm 0.358$ & $8.275 \pm 0.226$ & 0.375 \\
\hline UST $(\%)^{\#}$ & $44(65)$ & $16(80)$ & $28(58)$ & 0.091 \\
\hline Terminal (\%) ${ }^{\#}$ & $24(35)$ & $4(20)$ & $20(42)$ & 0.091 \\
\hline No symptoms during test (\%) & $30(44)$ & $12(60)$ & $18(37)$ & 0.091 \\
\hline Typical angina during test (\%) & $3(4)$ & $0(0)$ & $3(100)$ & 0.259 \\
\hline Atypical angina during test $(\%)$ & $21(31)$ & $4(20)$ & $17(35)$ & 0.216 \\
\hline Dyspnea during test $(\%)^{\#}$ & $31(46)$ & $8(40)$ & $23(48)$ & 0.557 \\
\hline Arrhythmia during test (\%) ${ }^{\#}$ & $34(50)$ & $8(40)$ & $26(54)$ & 0.294 \\
\hline MPHR, bpm & $161.088 \pm 1.033$ & $160.100 \pm 1.799$ & $161.500 \pm 1.264$ & 0.541 \\
\hline Exercise load & $2.838 \pm 0.065$ & $2.900 \pm 0.124$ & $2.813 \pm 0.077$ & 0.543 \\
\hline Peak HR, bpm & $151.397 \pm 2.102$ & $158.200 \pm 3.911$ & $148.563 \pm 2.401$ & 0.036 \\
\hline MPHR achieved, \% & $93.441 \pm 1.217$ & $98.350 \pm 2.240$ & $91.396 \pm 1.358$ & 0.008 \\
\hline maximum RPP & $248.657 \pm 8.871$ & $278.700 \pm 15.027$ & $236.640 \pm 9.775$ & 0.027 \\
\hline Exercise time, min & $6.765 \pm 0.185$ & $6.969 \pm 0.321$ & $6.679 \pm 0.227$ & 0.478 \\
\hline Pre-TET Resting SBP, mm Hg & $131.735 \pm 2.200$ & $130.300 \pm 3.220$ & $132.333 \pm 2.830$ & 0.677 \\
\hline Pre-TET Resting DBP, mm Hg & $77.500 \pm 1.384$ & $78.500 \pm 2.124$ & $77.083 \pm 1.755$ & 0.644 \\
\hline Post-TET Resting SBP, mm Hg & $130.515 \pm 1.816$ & $131.900 \pm 3.029$ & $129.938 \pm 2.257$ & 0.626 \\
\hline Post-TET Resting DBP, mm Hg & $79.868 \pm 1.282$ & $79.400 \pm 2.156$ & $80.063 \pm 1.590$ & 0.816 \\
\hline Peak SBP, bpm & $184.838 \pm 3.151$ & $185.050 \pm 5.783$ & $184.750 \pm 3.800$ & 0.966 \\
\hline Peak DBP, bpm & $82.941 \pm 1.692$ & $85.450 \pm 2.800$ & $81.896 \pm 2.094$ & 0.342 \\
\hline \multicolumn{5}{|l|}{ Duke Treadmill Score } \\
\hline Group $1(\geq 5), n(\%)$ & $12(17.6)$ & $4(33)$ & $8(67)$ & $<0.001$ \\
\hline Group $2(-10$ to $<5), \mathrm{n}(\%)$ & $48(70.6)$ & $6(12)$ & $42(88)$ & $<0.001$ \\
\hline Group $3(\leq-11), \mathrm{n}(\%)$ & $8(11.8)$ & $0(0)$ & $8(100)$ & $<0.001$ \\
\hline
\end{tabular}

Statistical comparisons of repeated multiple comparisons were carried out with Independent samples t-test. ${ }^{\circledR}$ Patients after PCI who had T-wave changes. "Statistical comparisons of repeated multiple comparisons were carried out with Chi-square test. Values are n (\%) or mean \pm SEM. Typical angina individuals were with complaints of angina, exertional chest discomfort, after that it was substernal and relieved with rest or nitroglycerin. 


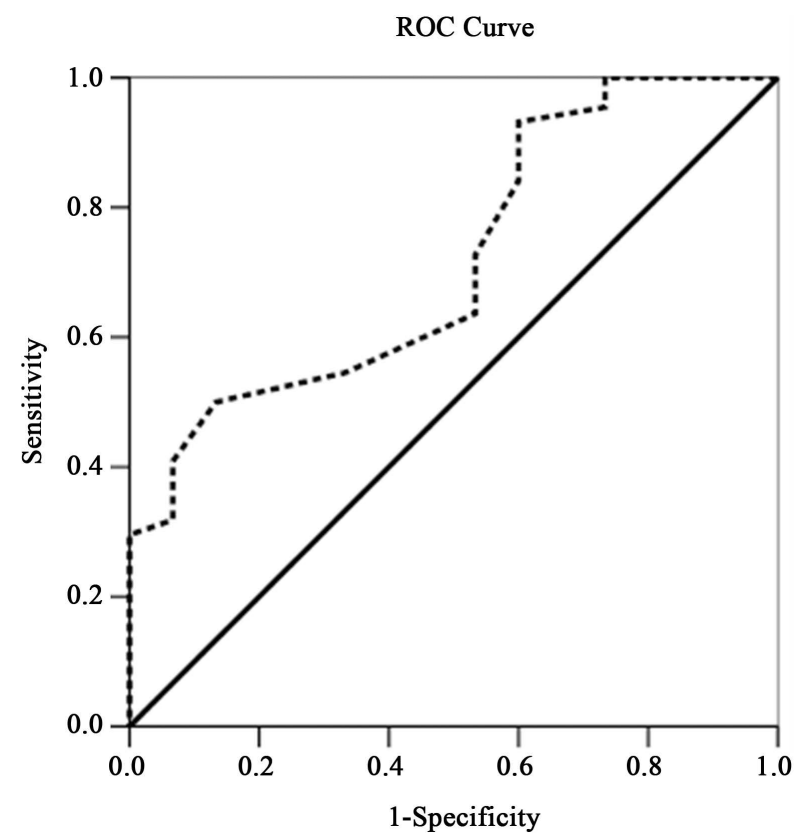

Figure 1. ROC curve were described of HbA1c for CHD diagnosis. The AUC was $0.718(0.500,0.867), P<0.05$. The best cutoff value was $5.85 \%$ which made a largest sum of sensitivity and specificity $(0.500,0.867$, and 1.367 , respectively).

Table 2. Determinations and tests of biochemical indexes.

\begin{tabular}{|c|c|c|c|}
\hline & CAG & $\operatorname{TET}(+, \pm)$ & TET (-) \\
\hline \multirow{2}{*}{ BUN (mmol/L) } & - & $347.692 \pm 27.927$ & $397.000 \pm 38.142$ \\
\hline & + & $381.081 \pm 19.537$ & $449.667 \pm 48.257$ \\
\hline \multirow{2}{*}{$\mathrm{CHL}(\mathrm{mmol} / \mathrm{L})$} & - & $4.507 \pm 0.303$ & $4.646 \pm 0.334$ \\
\hline & + & $4.838 \pm 0.228$ & $4.083 \pm 0.444$ \\
\hline \multirow{2}{*}{$\mathrm{CK}(\mathrm{mmol} / \mathrm{L})$} & - & $91.231 \pm 11.184$ & $124.571 \pm 11.820$ \\
\hline & + & $103.028 \pm 6.930$ & $222.429 \pm 123.171$ \\
\hline \multirow{2}{*}{ CKMB $(\mathrm{mmol} / \mathrm{L})$} & - & $14.077 \pm 1.059$ & $17.429 \pm 1.131$ \\
\hline & + & $17.028 \pm 1.799$ & $24.000 \pm 6.448$ \\
\hline \multirow{2}{*}{ HDL (mmol/L) } & - & $1.290 \pm 0.085$ & $1.143 \pm 0.133$ \\
\hline & + & $1.272 \pm 0.068$ & $1.089 \pm 0.061$ \\
\hline \multirow{2}{*}{ LDH1 (mmol/L) } & - & $34.385 \pm 2.168$ & $33.571 \pm 2.339$ \\
\hline & + & $44.353 \pm 9.767$ & $74.000 \pm 30.232$ \\
\hline \multirow{2}{*}{$\mathrm{LDL}(\mathrm{mmol} / \mathrm{L})$} & - & $2.278 \pm 0.232$ & $2.380 \pm 0.404$ \\
\hline & + & $2.935 \pm 0.171$ & $2.248 \pm 0.272$ \\
\hline \multirow{2}{*}{$\mathrm{TP}(\mathrm{mmol} / \mathrm{L})$} & - & $70.125 \pm 1.919$ & $68.514 \pm 2.862$ \\
\hline & + & $68.216 \pm 1.173$ & $69.288 \pm 2.002$ \\
\hline \multirow{2}{*}{ TG (mmol/L) } & - & $1.651 \pm 1.173$ & $1.754 \pm 0.221$ \\
\hline & + & $1.417 \pm 0.093$ & $1.686 \pm 0.309$ \\
\hline \multirow{2}{*}{ HbA1c (\%) } & - & $5.509 \pm 0.109^{*}$ & $5.450 \pm 0.273$ \\
\hline & + & $6.077 \pm 0.147^{*}$ & $6.078 \pm 0.267$ \\
\hline
\end{tabular}

+: positive; -: negative; \pm : inconclusive. *: significantly differences between CAG $(-)$ and CAG $(+)$ in TET $(+, \pm)$ group. 
Table 3. Comparison of different examinations for the 68 patients.

\begin{tabular}{ccccccc}
\hline Results & \multicolumn{2}{c}{ CAG } & TET & \multicolumn{2}{c}{ HbA1c } \\
\hline & $\mathrm{n}$ & $\%$ & $\mathrm{n}$ & $\%$ & $\mathrm{n}$ & $\%$ \\
+ & 48 & 0.705882353 & 38 & 0.558823529 & 29 & 0.426470588 \\
- & 20 & 0.294117647 & 16 & 0.235294118 & 30 & 0.441176471 \\
\hline
\end{tabular}

nosis, which were TET alone, HbA1c alone, and the combination of these two for either serial $(\mathrm{TET} \times \mathrm{HbA1c}$, both examinations positive could be presumed as positive) or parallel (TET + HbA1c, defined as either HbA1c or TET was positive) tests. For HbA1c, the best cutoff value used to estimate positive and negative patients was 5.85\%. As shown in Figures 2(a)-(d), compared with the gold standard diagnosis of CAG by ROC curve, the sensitivity values of TET alone, HbA1c alone, TET $\times$ HbA1c and TET + HbA1c were $60 \%, 55 \%, 36 \%$ and $80 \%$, respectively; and the specificity values of them were $60 \%, 67 \%, 89 \%$ and $31 \%$, respectively (Figure $2(\mathrm{e})$ ). These data demonstrated that all the patients who were negative in both HbA1c and TET turned out to be CAG negative [only $2(10 \%)$ of negative TET + HbA1c patient in CAG positive which was not shown], which meant that the combination of these two alternative non-invasive examinations could potentially represent of invasive CAG for ruling out CHD diagnosis.

\section{Discussion}

This study was about the analyses of generally acknowledged serum biochemical indexes in the suspected CHD patients. For each of them, serum assays were performed. TET which was an examination could assist CHD diagnosis and might arouse myocardial ischemia to make us study the combination TET and results of those biochemical indexes [9]. However, we did not find any significant changes among positive $(+)$, inconclusive $( \pm)$ and negative $(-)$ TET patients. We presumed that maybe some other biochemical indexes did rise, but the single time point of blood drawing was too inappropriate to capture that.

After the biochemical indexes were compared between CAG positive and CAG negative groups in positive TET $(+, \pm$ ) and negative TET $(-)$ groups, one significant difference was found: HbA1c levels in CAG positive group were higher than those in CAG negative group. In other words, HbA1c were significantly different between CHD patients and non-CHD people, which made it a potential biomarker to help diagnose in suspected CHD. ROC curve was used to testify this hypothesis, which showed that HbA1c had effective diagnosis for CHD and the best cutoff value for identifying positive or negative was $5.85 \%$.

Thus besides CAG, we had another four detective diagnosis for CHD, which were TET, HbA1c, and combination of both as serial and parallel tests. As a result, we found that the parallel tests had higher sensitivity of CHD diagnosis (80\%) than that of the TET examination (60\%). This has important clinical meanings. Although CAG was a golden standard diagnosis of CHD, it was an invasive procedure. Since both TET and HbA1c are non-invasive, cheap, high sensitivities and accessible, they can be used as screen examinations in suspected CHD patients. Although the specificities of tests dropped down $29 \%$ for parallel tests, it was still acceptable fact that parallel tests were efficient methods to diagnose CHD. As long as the serious consequence of missed diagnosis for CHD, high sensitivity was more important than low specificity. However, the serial tests can be compensated for low specificity of parallel tests, for the specificity was as high as $89 \%$.

In this study, we also found that all the persons with negative TET and HbA1c results were also $90 \%$ negative CAG, for this group of people, the non-invasive serial tests could stand CAG for ruling out CHD diagnosis, decreasing lots of unnecessary pains and risks.

To summarize, in our study, the HbA1c (\%) of CHD patients were higher than those of non-CHD persons. By means of ROC curve, it was found to have diagnostic effect of CHD, and could obviously enhance the sensitivity of examinations in combination with TET as parallel tests. And because of the identical results, the latter strategy could potentially stand invasive CAG for the diagnosis of CHD. However, considering the number of patients in our study is relatively limited, and so far no other similar studies were reported, further researches and evidence are warranted to validate the findings in this paper. 


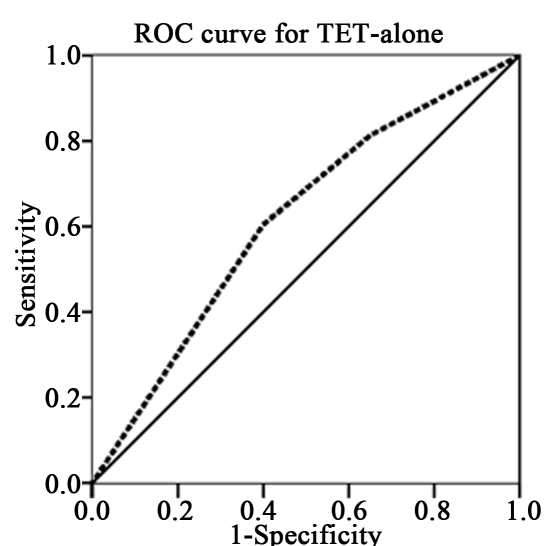

(a)

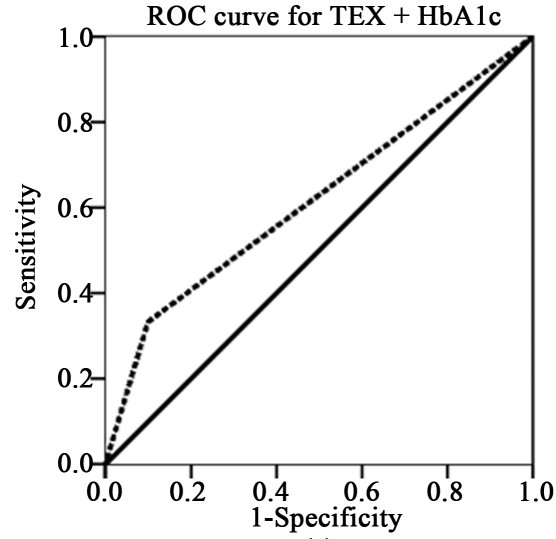

(c)

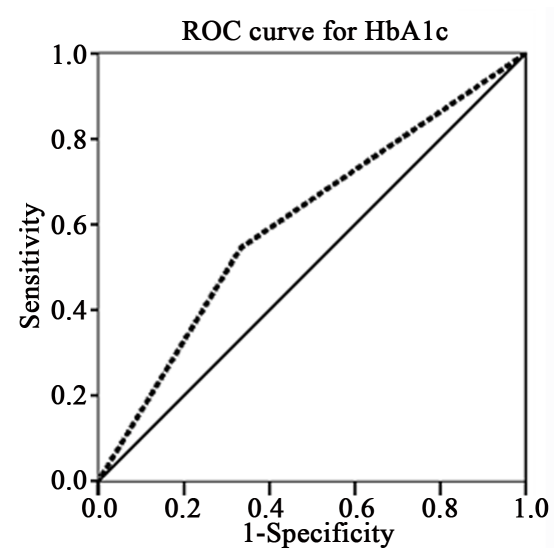

(b)

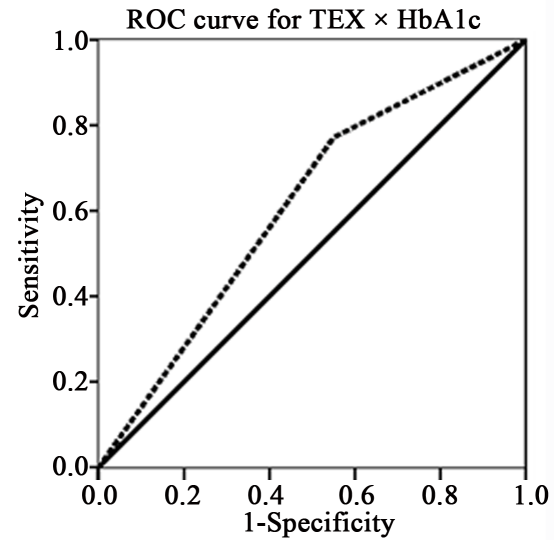

(d)

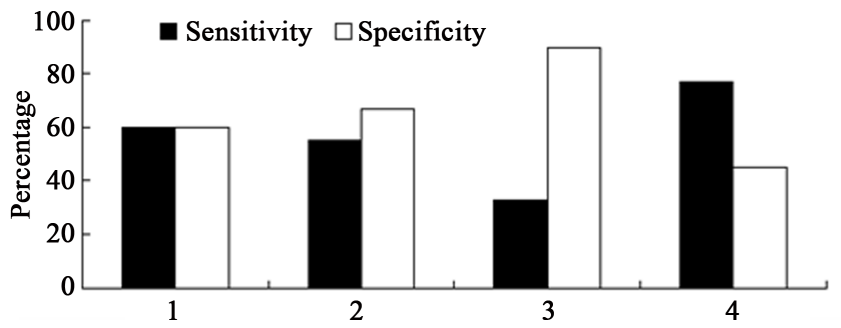

(e)

Figure 2. Comparison of different detections for CHD diagnosis. (a) ROC curve for TET results; (b) ROC curve for HbA1c results; (c) ROC curve for TET $\times$ HbA1c results; (d) ROC curve for TET + HbA1c results; (e) 1: TET; 2: HbA1c; 3: TET $\times$ HbA1c; 4: TET + HbA1c. TET $\times$ HbA1c represents serial tests of which positive is defined as both are positive, whereas TET + HbA1c stands for the parallel tests of which positive is defined as either TET or HbA1c is positive.

\section{Conclusion}

We found that HbA1c combined with TET obviously enhanced the sensitivity of examinations. All the patients who were negative in both HbA1c and TET tests turned out to be 90\% CAG negative, which meant that the combination might stand invasive examination of CAG for CHD diagnosis. Further studies in multi-center investigation will be expected to validate the findings.

\section{Acknowledgements}

We sincerely thank Zicheng Li and Jitian Xia for their generous assistance in manuscript preparation and clinical laboratory for the biochemical indexes in detection. 


\section{Funding}

This work was supported by Grants from Science and Information Technology of Guangzhou (No. 40108003), the Fundamental Research Funds for Central Universities (No. 21611333). The funders had no role in study design, data collection and analysis, decision to publish, or preparation of the manuscript.

\section{Ethical Approval}

The study received the First Affiliated Hospital of Jinan University Ethic Committee approval. Informed consent was obtained from all 68 patients.

\section{Conflicts of Interest}

The authors have declared that no competing interests exist.

\section{References}

[1] Zhao, W., Katzmarzyk, P.T., Horswell, R., Wang, Y., Johnson, J., et al. (2014) HbA1c and Coronary Heart Disease Risk among Diabetic Patients. Diabetes Care, 37, 428-435. http://dx.doi.org/10.2337/dc13-1525

[2] Kachintorn, U. (2005) How Do We Define Non-Cardiac Chest Pain? Journal of Gastroenterology and Hepatology, 20, S2-S5. http://dx.doi.org/10.1111/j.1440-1746.2005.04164.x

[3] Thorley, P.J., Bloomer, T.N., Sheard, K.L. and Sivananthan, U.M. (1996) The Use of GTN to Improve the Detection of Ischaemic Myocardium Using 99Tcm-Tetrofosmin. Nuclear Medicine Communications, 17, 669-674. http://dx.doi.org/10.1097/00006231-199608000-00004

[4] Gregoratos, G. (1998) Clinical Presentation of Coronary Artery Disease in the Elderly: How Does It Differ from the Younger Population? The American Journal of Geriatric Cardiology, 7, 35-40.

[5] Lear, S.A., Brozic, A., Myers, J.N., Ignaszewski, A. (1999) Exercise Stress Testing. An Overview of Current Guidelines. Sports Medicine, 27, 285-312. http://dx.doi.org/10.2165/00007256-199927050-00002

[6] Wierzbowska, K., Kurpesa, M., Peruga, J., Drozdz, J., Krzeminska-Pakula, M. and Kasprzak, J. (2002) Role of the right chest lead in improving the accuracy of myocardial ischemia detection. Przeglad Lekarski, 59, 684-686.

[7] Zhu, Z.H., Yan, Y., Wang, Q.B., Qian, J.Y., Ge, J.B. (2010) Analysis of Serum Cardiac Biomarkers and Treadmill Exercise Test-Electrocardiogram for the Diagnosis of Coronary Heart Disease in Suspected Patients. Acta Biochimica et Biophysica Sinica, 42, 39-44. http://dx.doi.org/10.1093/abbs/gmp103

[8] Kurata, A., Kono, A., Sakamoto, T., Kido, T., Mochizuki, T., et al. (2014) Quantification of the Myocardial Area at Risk Using Coronary CT Angiography and Voronoi Algorithm-Based Myocardial Segmentation. European Radiology, 25, 49-57

[9] Sun, J.L., Gao, G.L., Zhao, Y., Gao, Y.L., Xiong, L.J., et al. (2013) Elderly Patients and Coronary Heart Disease on Response to Treadmill Exercise Test. Cell Biochemistry and Biophysics, 67, 965-968. http://dx.doi.org/10.1007/s12013-013-9590-z 